\title{
Optimizing the spatial resolution of ChanneIrhodopsin-2 activation
}

\author{
Philipp Schoenenberger, Åsa Grunditz, Tobias Rose and Thomas G. Oertner* \\ Friedrich Miescher Institute, Maulbeerstrasse 66, WRO-1066.4.04, 4058 Basel, Switzerland \\ (*author for correspondence; e-mail: thomas.oertner@fmi.ch)
}

Received 19 February 2008; Accepted 29 May 2008

(c) Springer Science+Business Media, LLC 2008

\begin{abstract}
Over the past few years, the light-gated cation channel Channelrhodopsin-2 (ChR2) has seen a remarkable diversity of applications in neuroscience. However, commonly used wide-field illumination provides poor spatial selectivity for cell stimulation. We explored the potential of focal laser illumination to map photocurrents of individual neurons in sparsely transfected hippocampal slice cultures. Interestingly, the best spatial resolution of photocurrent induction was obtained at the lowest laser power. By adjusting the light intensity to a neuron's spike threshold, we were able to trigger action potentials with a spatial selectivity of less than $30 \mu \mathrm{m}$. Experiments with dissociated hippocampal cells suggested that the main factor limiting the spatial resolution was ChR2 current density rather than scattering of the excitation light. We conclude that subcellular resolution can be achieved only in cells with a high ChR2 expression level and that future improved variants of ChR2 are likely to extend the spatial resolution of photocurrent induction to the level of single dendrites.
\end{abstract}

\section{Introduction}

The light-gated channel Channelrhodopsin-2 (ChR2) is a versatile tool for the controlled activation of neurons. The temporal precision of lightinduced action potential generation can be as good as $1 \mathrm{~ms}$ (Boyden et al., 2005), but the spatial resolution limit of ChR2 activation has not been systematically explored. Spatial specificity of light stimulation can be achieved by two methods: Selective expression of ChR2 in a subset of cells (Adamantidis et al., 2007), or restriction of the illumination to a small region of tissue or even to a single cell (Wang et al., 2007). Different strategies for introducing ChR2 into neurons have been used:

Electronic supplementary material The online version of this article (doi:10.1007/s11068-008-9025-8) contains supplementary material, which is available to authorized users.
Viral transfection (Adamantidis et al., 2007; Aravanis et al., 2007; Zhang et al., 2008), in utero electroporation (Petreanu et al., 2007; Huber et al., 2008), and the generation of Thy 1 transgenic mice (Arenkiel et al., 2007; Wang et al., 2007). Most of these techniques produce a relatively high density of ChR2 expressing cells, and it is not clear whether individual cells can be activated by focused illumination under these conditions.

Here we use particle-mediated gene transfer to achieve a low density of transfected neurons in organotypic slice cultures, allowing us to explore the spatial resolution limit of ChR2 activation in detail. We used a focused laser beam to generate current and spike maps of ChR2 expressing cells. Surprisingly, spatial resolution improved with reduced laser power, which made it possible to map the position of a single cell and the orientation of the main apical dendrite, but not the location of fine dendrites. Degradation of the laser focus due to 
light scattering in the tissue was not a limiting factor in our experiments. Furthermore, we show that the previously described after-depolarization (Zhang and Oertner, 2007) is much reduced with focused illumination, indicating that a blue laser might be the ideal light source to mimic the properties of naturally occurring action potentials.

\section{Results}

\section{Characterization of laser-induced photocurrents}

In order to measure the spatial selectivity of ChR2 activation, we measured laser-induced photocurrents in pyramidal neurons voltage-clamped at $-65 \mathrm{mV}$. Recordings were performed in ACSF containing TTX and NBQX to isolate light-induced currents. Arc lamp stimulation with blue excitation light (EGFP filterset, 470/40 exciter) induced large photocurrents ( $3.3 \pm 0.9 \mathrm{nA}, n=5$ cells). For laser stimulation at $488 \mathrm{~nm}$, the end of a singlemode optical fiber was imaged into the center of the visual field (see "Methods"). Scanning was achieved by computer-controlled movements of the motorized microscope stage on which recording chamber and patch-clamp manipulators were mounted. Compared to wide-field illumination, laser-evoked photocurrents were much smaller $(0.7 \pm 0.2 \mathrm{nA})$, even at high laser intensities (350 $\mu \mathrm{W}$ at the back aperture of the objective), indicating that only a small fraction of the total ChR2 present in the membrane was activated by the focused laser light (Fig. 1a). The relationship between laser power and current amplitude was linear below $10 \mu \mathrm{W}$ but sublinear for higher laser powers (Fig. 1b). The sublinear characteristic suggests that ChR2 became saturated within the laser focus. Fluorescence emitted from the YFP-label of ChR2 showed less saturation with increasing laser power (Fig. 1b) due to its much faster photocycle: A single fluorescent protein can emit more than 2000 photons/ms (Kubitscheck et al., 2000), whereas ChR2 takes several seconds to return to the ground state (Bamann et al., 2008). Therefore, YFP fluorescence is proportional to ChR2 currents only at very low light intensities.

Next we explored the spatial resolution of photocurrent generation. Pyramidal neurons were voltage-clamped and laser-induced currents were recorded while the cells were moved in $10 \mu \mathrm{m}$ steps along a line perpendicular to the orientation of the apical dendrite (Fig. 1c). The length of the scanned line was $200 \mu \mathrm{m}$ and the soma was positioned in the middle. Stimulation pulses were applied with intervals of $5 \mathrm{~s}$ to allow for recovery of ChR2-mediated currents. The photocurrents reached maximum amplitude when the soma was in the center of the laser spot and decreased with increasing distance from the laser beam (Fig. 1c). Surprisingly, at laser powers of $15 \mu \mathrm{W}$ or more, large photocurrents were induced even at a distances above $100 \mu \mathrm{m}$ lateral from the soma (Fig. 1d). As a measure for the spatial resolution of photocurrent generation, we used the full width at half maximum current (FWHM) for different laser intensities (Fig. 1e). Interestingly, spatial resolution was best at the lowest stimulation intensity $(\mathrm{FWHM}=35 \mu \mathrm{m}$ at $0.7 \mu \mathrm{W}, 115 \mu \mathrm{m}$ at $15 \mu \mathrm{W}$, $\sim 180 \mu \mathrm{m}$ at $60 \mu \mathrm{W}, n=3$ ). Taken together, our data indicate that laser illumination activates a small fraction of the total membrane-bound ChR2, and that the spatial resolution of laser stimulation depends on laser intensity.

\section{Laser intensity and illuminated area}

In order to better understand the effect of laser intensity on spatial resolution, we made use of the fact that both the chromophore (all-trans retinal) and the YFP label of ChR2 are excited by the $488 \mathrm{~nm}$ laser line. Therefore, at low laser power, YFP fluorescence can be used as a reporter of the spatial extent of ChR2 activation. In the center of the laser beam, however, the relationship between YFP fluorescence and photocurrent will not be linear due to stronger saturation of ChR2 (Fig. 1b). We focused the laser on the soma of a ChR2-YFP expressing cell and captured YFP fluorescence excited at different laser intensities (Fig. 2a). The membrane area with detectable YFP fluorescence increased as a function of laser intensity, which is best seen in intensity profiles measured across the soma (Fig. 2b). With $350 \mu \mathrm{W}$ laser power, we detected fluorescence from dendrites up to $80 \mu \mathrm{m}$ from the laser focus (data not shown). To measure the intensity profile of the excitation beam, we imaged the fluorescence excited in a thin film of fluorescein solution. The profile was symmetric with $\mathrm{FWHM}=2.7 \mu \mathrm{m}$ (independent of laser power in the tested range of $10-60 \mu \mathrm{W}$ ) and long tails (Fig. 2c). From these findings, we conclude that the membrane area generating ChR2-mediated photocurrents increases as a function of laser intensity. 

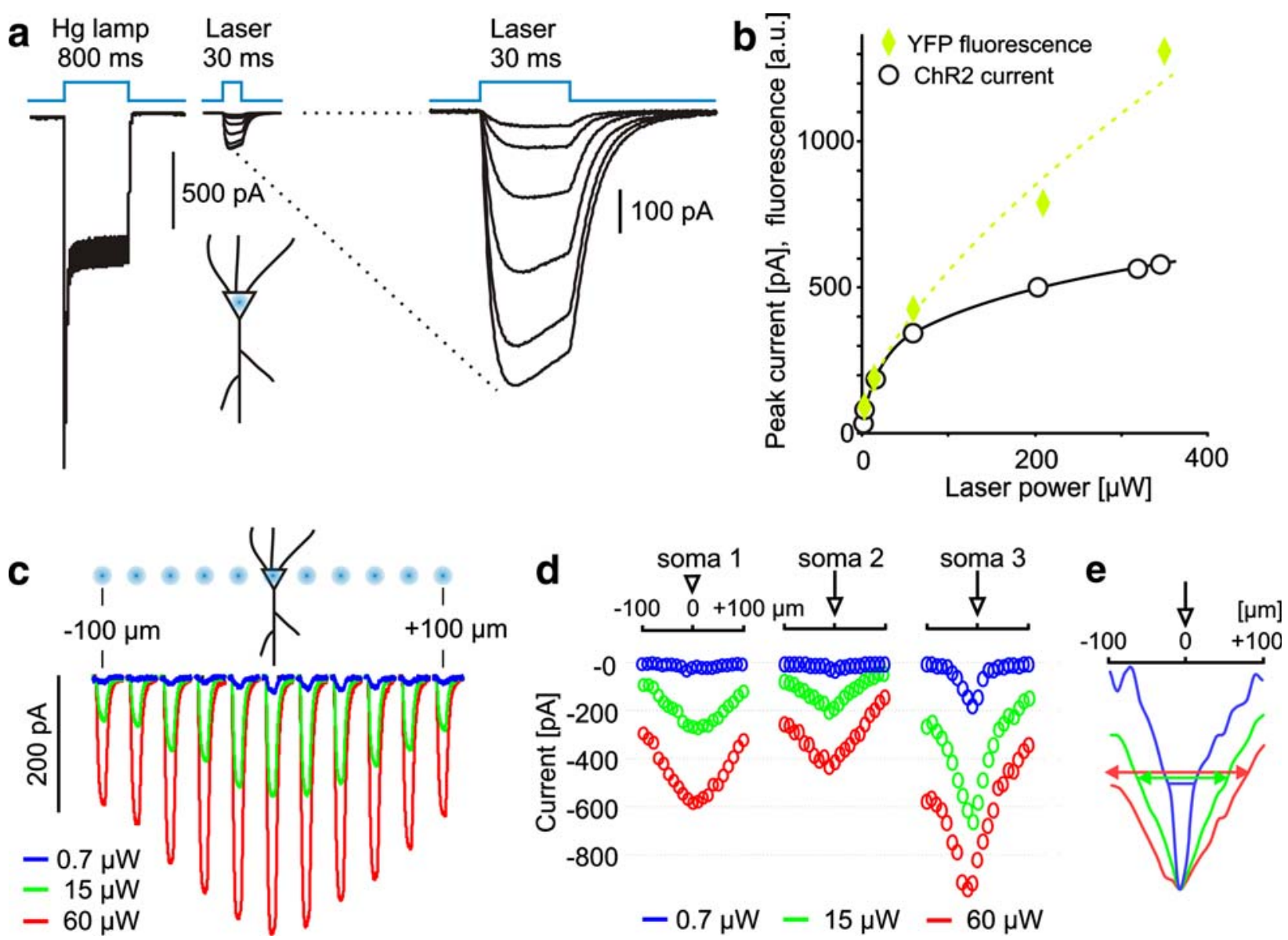

Fig. 1. Induction of photocurrents by laser stimulation of ChR2. (a) Comparison of ChR2 currents during wide-field stimulation (Hg arc lamp, $0.8 \mathrm{~mW}$ ) and focused laser stimulation at the soma (note different time scales). (b) Relationship between photocurrent amplitude and laser intensity shows strong saturation at laser powers above $20 \mu \mathrm{W}$. Integrated fluorescence of ChR2-YFP (green diamonds, arbitrary units) shows less saturation for the same range of laser intensities. (c) Currents evoked by a series of laser pulses with $10 \mu \mathrm{m}$ steps. For clarity, only every second current trace and schematic laser spot are shown. (d) Peak photocurrent amplitudes with respect to laser position. Three different cells were stimulated as in (c) at 3 laser intensities. (e) Peak-normalized photocurrents ( $n=3$ cells). Full-width at half maximum activation $(35,115,180 \mu \mathrm{m})$ depends on laser intensity.

In the laser focus, the photocycle of ChR2 is likely to become saturated, thus the current will not scale with laser power at medium to high intensities. The recruitment of more and more distal ChR2 channels with increasing laser power explains the apparent loss in spatial resolution (Fig. 1c-e).

\section{Two-dimensional current mapping in slice culture and dissociated neurons}

Local photocurrents could potentially be used as a tool to investigate electrotonic or active signal propagation in individual dendrites. To explore this possibility, we stimulated voltage-clamped cells in a grid pattern (30 and $10 \mu \mathrm{m}$ step size) at different laser intensities (Fig. 3a). We found that current amplitudes were highly reproducible, revealed the location of the soma and the orientation of the main dendrite, but did not resolve higher order dendrites (Fig. 3b). As an operational definition of spatial resolution, we determined the FWHM of a Gaussian fit in the direction of the steepest drop of the currents from the center of the soma (Fig. 3c). This strategy minimizes the influence of cell morphology (e.g., apical dendrite) on the resolution measurement. For 

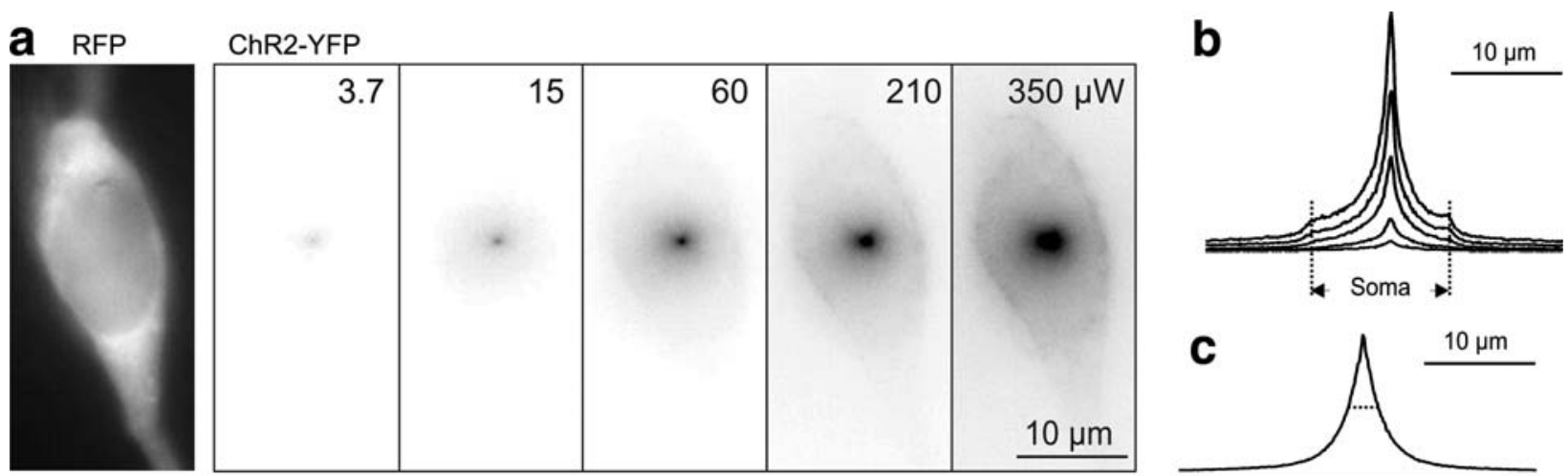

Fig. 2. High laser power leads to excitation of ChR2-YFP molecules outside the focal spot. (a) Left: RFP image showing soma of transfected cell. Right: ChR2-YFP signal excited with different laser intensities (0.3 s exposure). (b) YFP fluorescence intensity profiles across the soma through the center of the laser spot. (c) Laser-excited fluorescence intensity profile measured in thin film of fluorescein solution $(\mathrm{FWHM}=2.7 \mu \mathrm{m}$, laser power $=60 \mu \mathrm{W})$.

the cell analyzed in Fig. 3, resolution was $41 \mu \mathrm{m}$ at $4 \mu \mathrm{W}, 69 \mu \mathrm{m}$ at $60 \mu \mathrm{W}$, and $114 \mu \mathrm{m}$ at $260 \mu \mathrm{W}$ laser power. At the lowest laser power tested, spatial resolution could be further improved by sampling in $10 \mu \mathrm{m}$ steps $(28 \mu \mathrm{m}$ at $4 \mu \mathrm{W}$, Fig. $3 \mathrm{~b}$, small square map). For $60 \mu \mathrm{W}$ laser power, $10 \mu \mathrm{m}$ steps did not improve resolution (63 $\mu \mathrm{m}$ FWHM). Similar results were obtained in five strongly expressing cells.

At the low laser powers that were best for mapping, somatic photocurrents were very small $(<140$ pA, Fig. 3d). Therefore, one important factor that limits the resolution of current maps is the noise floor of the electrophysiological recordings. Another unfavorable effect is the scattering of blue excitation light in the organotypic cultures. We wanted to test if the spatial resolution of ChR2 activation could be improved in isolated cells. For this purpose, we prepared low-density hippocampal cell cultures co-transfected with ChR2-YFP and RFP. Compared to cells in organotypic cultures, dissociated cells produced smaller photocurrents (Fig. 3e). The spatial resolution of current mapping was comparable to the resolution in organotypic slices at the same laser power $(n=4)$, indicating that light scattering was not a limiting factor when mapping cells in organotypic slice cultures.

\section{Spatial resolution of action potential triggering by local ChR2 activation}

The findings above suggested the possibility to spike ChR2-expressing cells with high spatial selectivity using low stimulation intensities. We investigated this possibility using cell-attached recordings and $10 \mathrm{~ms}$ laser pulses. At laser intensities just above threshold (typically $70-100 \mu \mathrm{W}$ ), action potentials (APs) were elicited only when the laser was exactly centered on the soma (Fig. 4a). A modest increase in laser power immediately decreased the spatial selectivity, since now APs were also triggered along the main trunk of the apical dendrite. Similar results were obtained using whole-cell current clamp recordings (Fig. 4b), confirming the inverse relationship between laser power and spatial resolution. At laser powers above $300 \mu \mathrm{W}$, spatial specificity was completely lost within a $100 \mu \mathrm{m}$ radius in most cells (Supp. Fig. 1). In cells with lower ChR2 expression levels, high laser powers, or longer laser pulses were needed to reach spike threshold. Consequently, spatial resolution in these cells was very poor, as action potentials were generated at distances above $100 \mu \mathrm{m}$ from the soma $(n=4$, data not shown). Taken together, these results show that ChR2-mediated action potentials can be triggered with high spatial selectivity using low stimulation intensities, and that the expression level of ChR2 limits the spatial resolution.

With increasing laser power, we noticed a pronounced after-depolarization appearing in the wake of the action potential (Fig. 4c, blue traces). This effect was not seen at laser intensities just above threshold (Fig. 4c, green traces) or if laser illumination was applied to the axonal arborization distal from the soma, generating an antidromic spike with a delay of 7-12 ms (Fig. 4c, black traces). After-depolarization following wide-field 
a

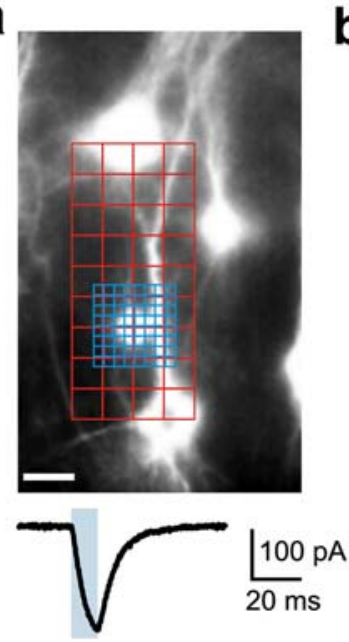

b

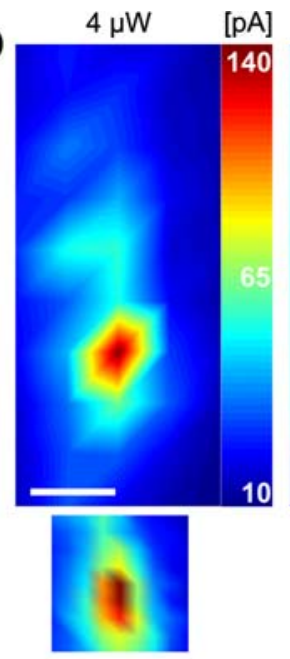

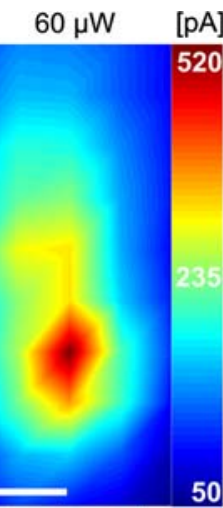

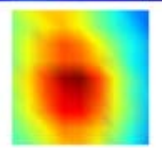

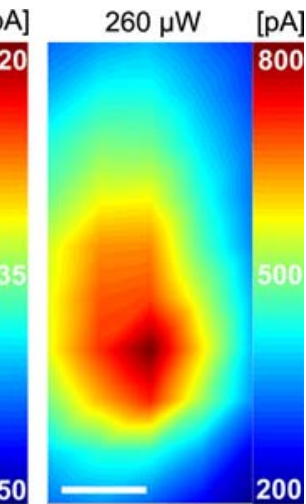

C
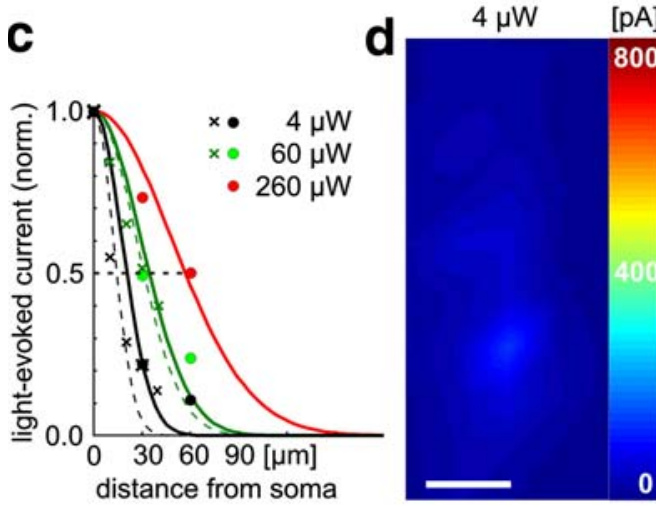

e

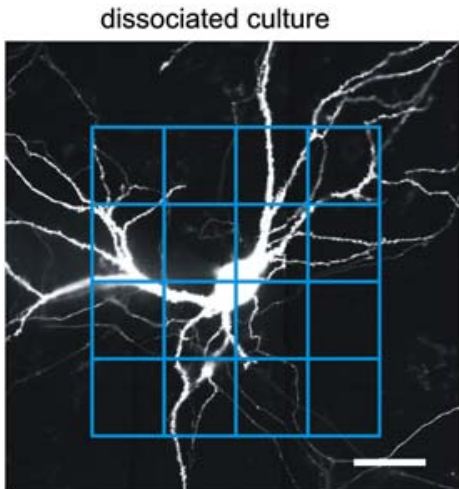

$60 \mu \mathrm{W}$

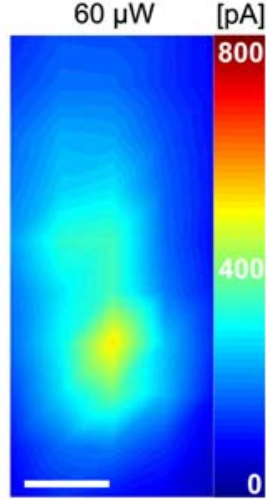

$210 \mu \mathrm{W}$

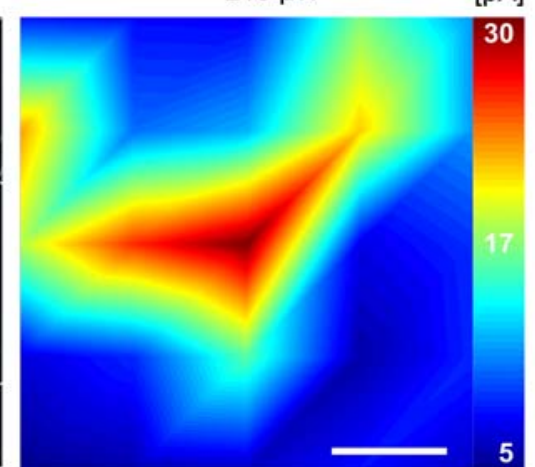

Fig. 3. Resolution of current maps depends on laser power. (a) Fluorescence image of ChR2 transfected cells in entorhinal cortex. The stage was moved relative to the laser focus in $30 \mu \mathrm{m}$ (red grid) and $10 \mu \mathrm{m}$ (blue grid) steps. Below: Example trace, $10 \mathrm{~ms}$ laser pulse. (b) Interpolated current maps for 3 different laser intensities for 30 and $10 \mu \mathrm{m}$ grid (lower maps). Maps are scaled to min/max current, $30 \mu \mathrm{m}$ and corresponding $10 \mu \mathrm{m}$ maps have identical scaling. Scale bars: $50 \mu \mathrm{m}$. Maps are averages of two repetitions. (c) Gaussian fits to the current drop away from the soma (best direction) show influence of laser power on resolution. $30 \mu \mathrm{m}$ Grid: circles, solid line fit, FWHM $=41$, 69, $114 \mu \mathrm{m}$. $10 \mu \mathrm{m}$ Grid: crosses, dashed line fit, FWHM $=28,63 \mu \mathrm{m}$. (d) Same data as in b, uniform scaling. (e) Fluorescence image of ChR2-transfected cell in dissociated hippocampal cell culture. Stimulation grid: $50 \mu \mathrm{m}$ steps, $210 \mu \mathrm{W}$ laser power. Right: Interpolated current map (average of 3 repetitions). Spatial resolution: $108 \mu \mathrm{m}$ FWHM. 
a

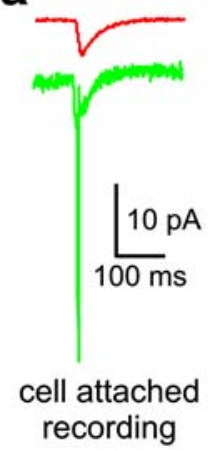

b

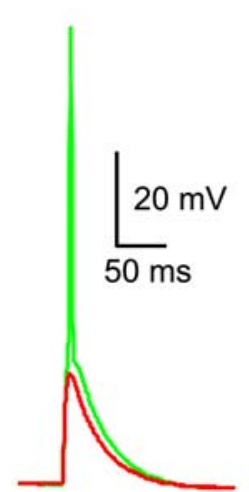

whole cell recording
$70 \mu \mathrm{W}$

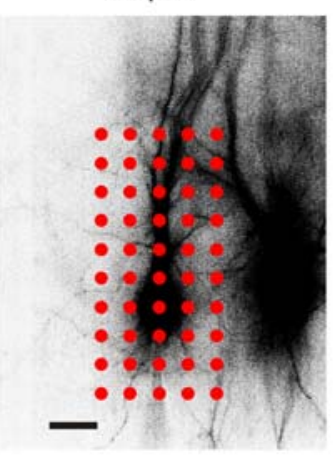

$70 \mu \mathrm{W}$
$90 \mu \mathrm{W}$
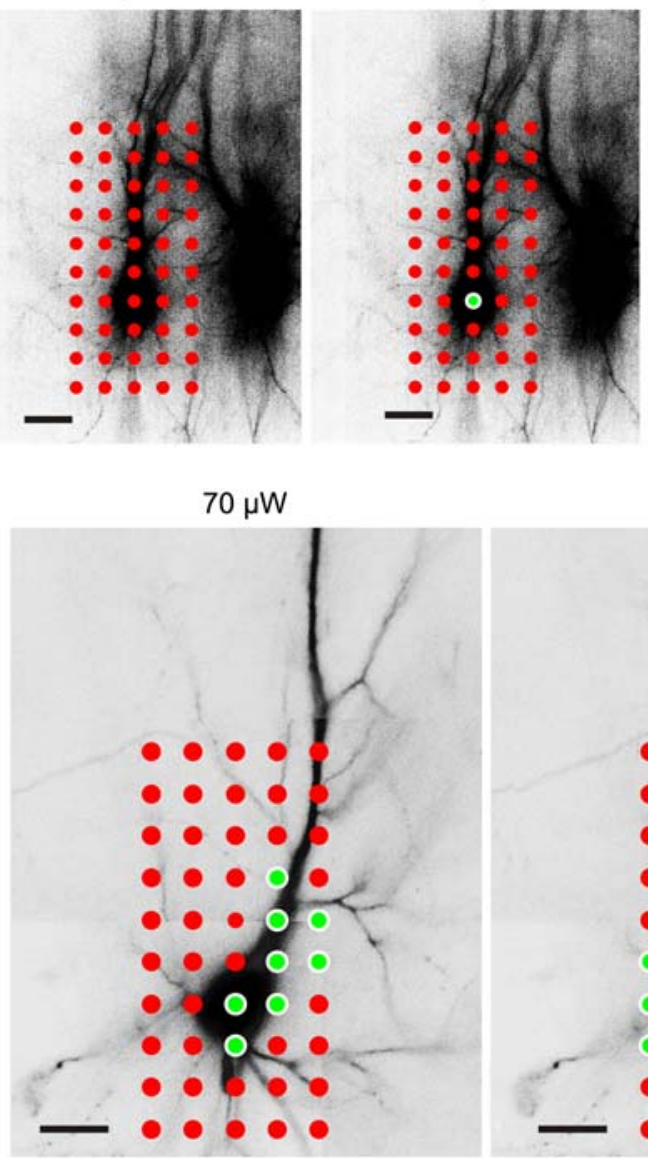

$150 \mu \mathrm{W}$

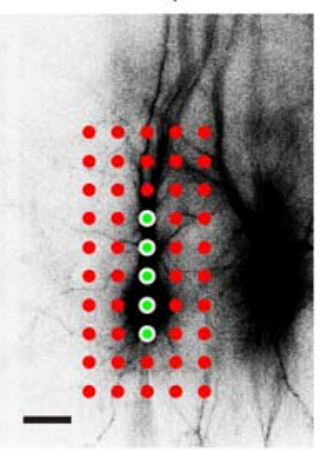

$90 \mu \mathrm{W}$
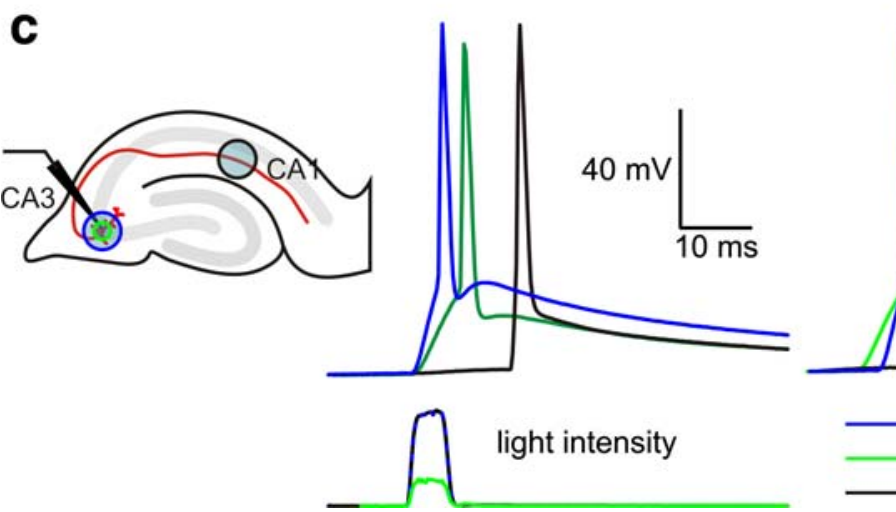

light intensity
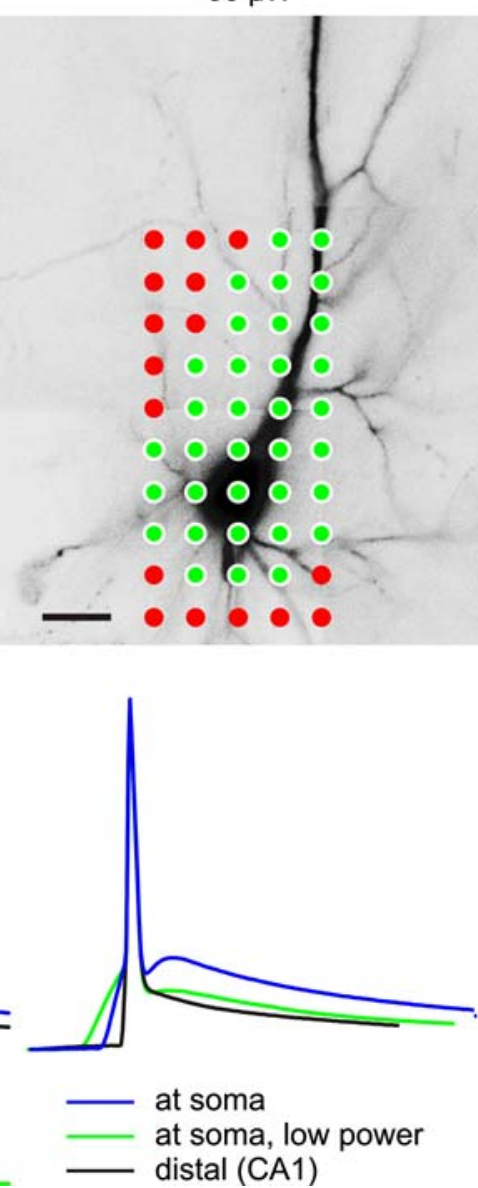

Fig. 4. Spatial resolution of action potential triggering. (a) Cell-attached recording of CA1 pyramidal cell. Red trace: subthreshold stimulation. Green trace: suprathreshold stimulation. Stimulation grid: $30 \mu \mathrm{m}$ step size. Scale bar: $50 \mu \mathrm{m}$. At laser powers just above threshold, spatial resolution was very good. Increasing laser power decreased spatial resolution of spike map (right). (b) Whole cell current clamp recording of CA1 pyramidal cell. High ChR2 expression level resulted in spike threshold below $70 \mu \mathrm{W}$ laser power. (c) Afterdepolarization in CA3 pyramidal cell is most pronounced if high intensity pulses are applied directly to the soma (blue trace). An identical laser pulse applied distally in CA1 triggers an antidromic spike with no apparent after-depolarization in the somatic current clamp recording (black trace). Right: Action potentials peak-aligned to compare after-depolarization. 
illumination leads to increased calcium influx and increases the probability of transmitter release at ChR2-expressing axonal boutons (Zhang and Oertner, 2007). We suggest that these effects could be largely avoided using focal illumination just above threshold.

\section{Discussion}

Here we show that the spatial resolution of $\mathrm{ChR} 2$ activation depends on the intensity of the laser beam. The soma represents only a small fraction of the total membrane of the cell, most surface area is contained in the extensive dendritic and axonal arborization. For this reason, wide-field illumination evokes 5- to 10-fold larger photocurrents than focused laser illumination at the soma (Fig. 1a). Due to the Gaussian intensity profile of a focused laser beam and scattering of excitation light, out-of-focus membrane becomes increasingly illuminated as the focal intensity is increased (Fig. 2), increasing the total photocurrent. This effect is counteracted by saturation of ChR2 activation in the center of the beam, resulting in a sublinear increase of total ChR2 current with increasing laser intensity (Fig. 1b). Since the 3-dimensional geometry of the cell increasingly determines ChR2 current amplitudes at high laser powers, the relationship between laser power and current amplitude cannot be predicted from first principles.

Comparing the shape of photocurrents at different laser intensities, we noted increasing inactivation with increasing laser power (Fig. 1a). In order to study the photocycle of light-gated channels, light pulses have to be either very brief or of saturating intensity to ensure synchronous activation of the entire channel population (Nagel et al., 2003; Bamann et al., 2008). Focused laser light, on the other hand, provides a spatial gradient of light intensities (Fig. 2). As a result, only channels in the focus will be activated synchronously, while more distal channels get activated at random time points, obscuring single channel kinetics in the total ChR2 current. At very low laser powers, the pool of channels in the ground state never gets depleted, resulting in an apparently non-inactivating photocurrent (Fig. 1a). In summary, stimulation with long and focused laser pulses is not a suitable method to analyze channel kinetics.
We used laser stimulation of ChR2 to generate current maps with subcellular resolution in organotypic and dissociated hippocampal cultures (Fig. 3). The best resolution ( $28 \mu \mathrm{m}$ FWHM) was obtained in a strongly expressing cell at very low laser power (Fig. 3c). Under these conditions, ChR2 currents were very small and a low-noise recording setup was required. Furthermore, resolution was slightly better with $10 \mu \mathrm{m}$ step size than with $30 \mu \mathrm{m}$ steps at the lowest laser powers, indicating that $30 \mu \mathrm{m}$ steps were undersampling the signal bandwidth. Therefore, for optimal (Nyquist) sampling, step size should be scaled to laser intensity.

In order to increase spatial resolution, higher ChR2 current densities would help to increase the signal-to-noise ratio at low laser power. This could be achieved by higher expression levels or increased single channel conductance of ChR2. We have not explored the possibility of nonGaussian beam profiles, which could help to reduce ChR2 saturation and inactivation in the center. Light scattering in the tissue seems to play a minor role for the relatively superficial transfected cells in organotypic slice cultures, but might be a key issue for in vivo applications (Aravanis et al., 2007; Arenkiel et al., 2007).

Under current clamp conditions, the sharp threshold of action potential generation leads to binarization of the depolarization map into a suprathreshold center and a subthreshold surround (Wang et al., 2007). We show that at laser intensities just above firing threshold, spatial selectivity can be better than $30 \mu \mathrm{m}$ (Fig. 4a). Since we relied on patch-clamp recordings to adjust the laser power, these calibration experiments might seem to be of little practical relevance. However, optical detection of single action potentials has been demonstrated using bulk loading of calcium- or voltage-sensitive dyes (Kasuga et al., 2003; Stosiek et al., 2003), and might also be possible with genetically encoded calcium indicators (Mao et al., 2008). A combination of optical stimulation and optical readout could be used to build a closed-loop stimulation system in which excitation intensity is automatically adjusted to be just above threshold for individual cells. Our experiments suggest that this would be an ideal strategy to optimize the specificity of optical stimulation and to compensate for cell-to-cell variability in ChR2 expression levels. 


\section{Methods}

\section{Cell culture and transfection}

Hippocampal slice cultures from rats (Sprague Dawley) were prepared at postnatal day $4-5$ as described (Stoppini et al., 1991), according to the rules of the Federal Veterinary Office of BaselStadt. After 7 days in culture, we used a Helios gene gun (Bio-Rad) to co-transfect individual cells with DNA encoding ChR2-YFP (K. Deisseroth) and tdimer2 (dimeric RFP, R.Y. Tsien), each subcloned into a neuron-specific (synapsin 1 ) expression vector. Dissociated hippocampal cultures were prepared as previously described (Fischer et al., 1998) and transfected using lipofectamine (Invitrogen).

\section{Electrophysiology and photostimulation}

The recording setup was based on a BX-51 microscope equipped with a LUMPlan $60 \times 0.9 N A$ water immersion objective (Olympus) and a cooled CCD camera (Sensicam QE). A secondary camera port was used to couple in $488 \mathrm{~nm}$ light (Melles Griot 543 argon ion laser) via single-mode optical fiber $(\mathrm{MFD}=3.2 \mu \mathrm{m})$. Acousto-optic modulator (AA Opto-Electronic) and position of the motorized stage (Sutter Instrument) were controlled by custom software written in MATLAB (The MathWorks). In order to avoid successive illumination of neighboring points, we used a step-back pattern (1-5-2-6-37...) for mapping. In order to monitor the time course of the light stimulus, we used a photomultiplier tube below the condenser to detect a small fraction of the blue excitation light transmitted through the preparation. Laser power was measured at the back aperture of the objective (LaserCheck, Coherent). For patch-clamp recordings, we used an Axopatch 200B amplifier (Molecular Devices) and MP-225 manipulators (Sutter Instrument). Experiments were conducted at room temperature 1-3 weeks after transfection. Artificial cerebrospinal fluid (ACSF) for slice cultures contained (in mM) $119 \mathrm{NaCl}, 26.2 \mathrm{NaHCO}_{3}, 11$ D-glucose, $2.5 \mathrm{KCl}, 4 \mathrm{MgCl}_{2}, 4 \mathrm{CaCl}_{2}, 1.0 \mathrm{NaH}_{2-}$ $\mathrm{PO}_{4}$. ACSF was complemented with $1 \mu \mathrm{M}$ TTX, $10 \mu \mathrm{M}$ NBQX for voltage-clamp experiments; $10 \mu \mathrm{M}$ bicuculline, $10 \mu \mathrm{M}$ dCPP, and $10 \mu \mathrm{M}$ NBQX in current-clamp experiments to block $\mathrm{GABA}_{\mathrm{A}}$, NMDA and AMPA receptors. Cell-attached recordings were performed under the same conditions as the current clamp experiments, pipettes contained $150 \mathrm{mM} \mathrm{NaCl}$. Glass pipettes for patch-clamp recordings were filled with intracellular solutions containing (in $\mathrm{mM}$ ): 135 potassium gluconate, 10 HEPES, $4 \mathrm{MgCl}_{2}$, $4 \mathrm{Na}_{2}$-ATP, $0.4 \mathrm{Na}-\mathrm{GTP}, 10 \mathrm{Na}_{2}-$ phosphocreatine, and 3 ascorbate for currentclamp experiments; 135 cesium methanesulfonate, 10 HEPES, $4 \mathrm{MgCl}_{2}$, $4 \mathrm{Na}_{2}$-ATP, $0.4 \mathrm{Na}$-GTP, 10 $\mathrm{Na}_{2}$-phosphocreatine, and 5 glutathione for voltageclamp. Recordings in dissociated cells were performed with solutions as described (Skeberdis et al., 2006).

\section{Data analysis}

Data were analyzed with custom software written in MATLAB. In order to generate color coded 2D maps, we used MATLAB's surf function with interpolated shading. Numerical values are given as mean $\pm S D$.

\section{Acknowledgments}

We thank Daniela Gerosa-Erni for excellent technical assistance, Nunu Mchedlishvili for software development, and Georg Nagel, Karl Deisseroth and Roger Y. Tsien for essential constructs. The work was supported by the Novartis Research Foundation.

\section{References}

Adamantidis, A. R., Zhang, F., Aravanis, A. M., Deisseroth, K., and de Lecea, L. (2007). Neural substrates of awakening probed with optogenetic control of hypocretin neurons. Nature 450, 420-424.

Aravanis, A. M., Wang, L. P., Zhang, F., Meltzer, L. A., Mogri, M. Z., Schneider, M. B., and Deisseroth, K. (2007). An optical neural interface: in vivo control of rodent motor cortex with integrated fiberoptic and optogenetic technology. J. Neural. Eng. 4, S143-S156.

Arenkiel, B. R., Peca, J., Davison, I. G., Feliciano, C., Deisseroth, K., Augustine, G. J., Ehlers, M. D., and Feng, G. (2007). In vivo light-induced activation of neural circuitry in transgenic mice expressing channelrhodopsin-2. Neuron 54, 205-218. 
Bamann, C., Kirsch, T., Nagel, G., and Bamberg, E. (2008). Spectral characteristics of the photocycle of channelrhodopsin-2 and its implication for channel function. J. Mol. Biol. 375, 686-694.

Boyden, E. S., Zhang, F., Bamberg, E., Nagel, G., and Deisseroth, K. (2005). Millisecond-timescale, genetically targeted optical control of neural activity. Nat. Neurosci. 8, 1263-1268.

Fischer, M., Kaech, S., Knutti, D., and Matus, A. (1998). Rapid actin-based plasticity in dendritic spines. Neuron 20, 847-854.

Huber, D., Petreanu, L., Ghitani, N., Ranade, S., Hromadka, T., Mainen, Z., and Svoboda, K. (2008). Sparse optical microstimulation in barrel cortex drives learned behaviour in freely moving mice. Nature 451, 61-64.

Kasuga, A., Enoki, R., Hashimoto, Y., Akiyama, H., Kawamura, Y., Inoue, M., Kudo, Y., and Miyakawa, H. (2003). Optical detection of dendritic spike initiation in hippocampal CA1 pyramidal neurons. Neuroscience 118, 899-907.

Kubitscheck, U., Kuckmann, O., Kues, T., and Peters, R. (2000). Imaging and tracking of single GFP molecules in solution. Biophys. J. 78, 2170-2179.

Mao, T., O'Connor, D. H., Scheuss, V., Nakai, J., and Svoboda, K. (2008). Characterization and subcellular targeting of GCaMP-type genetically-encoded calcium indicators. PLoS ONE 3, e1796.

Nagel, G., Szellas, T., Huhn, W., Kateriya, S., Adeishvili, N., Berthold, P., Ollig, D., Hegemann, P., and Bamberg, E. (2003). Channelrhodopsin-2, a directly light-gated cation- selective membrane channel. Proc. Natl. Acad. Sci. USA 100, 13940-13945.

Petreanu, L., Huber, D., Sobczyk, A., and Svoboda, K. (2007). Channelrhodopsin-2-assisted circuit mapping of long-range callosal projections. Nat. Neurosci. 10, 663-668.

Skeberdis, V. A., Chevaleyre, V., Lau, C. G., Goldberg, J. H., Pettit, D. L., Suadicani, S. O., Lin, Y., Bennett, M. V., Yuste, R., Castillo, P. E., and Zukin, R. S. (2006). Protein kinase A regulates calcium permeability of NMDA receptors. Nat. Neurosci. 9, 501-510.

Stoppini, L., Buchs, P. A., and Muller, D. (1991). A simple method for organotypic cultures of nervous tissue. J. Neurosci. Methods 37, 173-182.

Stosiek, C., Garaschuk, O., Holthoff, K., and Konnerth, A. (2003). In vivo two-photon calcium imaging of neuronal networks. Proc. Natl. Acad. Sci. USA 100, 7319-7324.

Wang, H., Peca, J., Matsuzaki, M., Matsuzaki, K., Noguchi, J., Qiu, L., Wang, D., Zhang, F., Boyden, E., Deisseroth, K., Kasai, H., Hall, W. C., Feng, G., and Augustine, G. J. (2007). High-speed mapping of synaptic connectivity using photostimulation in Channelrhodopsin-2 transgenic mice. Proc. Natl. Acad. Sci. USA 104, 8143-8148.

Zhang, Y. P. and Oertner, T. G. (2007). Optical induction of synaptic plasticity using a lightsensitive channel. Nat. Methods 4, 139-141.

Zhang, F., Prigge, M., Beyriere, F., Tsunoda, S. P., Mattis, J., Yizhar, O., Hegemann, P., and Deisseroth , K. (2008). Red-shifted optogenetic excitation: a tool for fast neural control derived from Volvox carteri. Nat. Neurosci. 11, 631-633. 